\title{
Involvement of the MiR-181b-5p/HMGB1 Pathway in Ang II-induced Phenotypic Transformation of Smooth Muscle Cells in Hypertension
}

\author{
Feng-Juan Li', Cheng-Long Zhang', Xiu-Ju Luo², Jun Peng,,,", Tian-Lun Yang1,* \\ ${ }^{1}$ Department of Cardiovascular Medicine, Xiangya Hospital, Central South University, Changsha 410008, China. \\ ${ }^{2}$ Department of Laboratory Medicine, Xiangya School of Medicine, Central South University, Changsha410013, \\ China. ${ }^{3}$ Department of Pharmacology, Xiangya School of Pharmaceutical Sciences, Central South University, \\ Changsha, China. ${ }^{4}$ Hunan Provincial Key Laboratory of Cardiovascular Research, Xiangya School of \\ Pharmaceutical Sciences, Central South University, Changsha 410078, China
}

[Received March 9, 2018; Revised April 28, 2018; Accepted May 10, 2018]

\begin{abstract}
Phenotypic transformation of vascular smooth muscle cells (VSMCs) contributes to vascular remodeling in hypertension. High mobility group box-1 (HMGB1) has been reported to be involved in several pathogenic processes including VSMC proliferation and migration. The present study was designed to determine the role of HMGB1 in VSMC phenotypic transformation in hypertension. First, we demonstrated that HMGB1 was elevated in a model of Ang II-induced VSMC phenotypic transformation, which showed down-regulation of contractile proteins and up-regulation of synthetic proteins. Knockdown of HMGB1 and losartan could block the phenotypic transformation. Next, we identified three potential miRNAs for upstream regulation of HMGB1 by bioinformatic analysis; only miR-181b-5p was significantly down-regulated in Ang II-treated cells. Co-treating the cells with miR-181b-5p mimics suppressed HMGB1 expression as well as the phenotypic transformation, migration, and proliferation. Furthermore, the luciferase reporter gene assay confirmed the direct interaction between miR-181b-5p and HMGB1. Finally, to extend these cell-based studies to clinical patients, we demonstrated that plasma miR-181b-5p levels were decreased, while Ang II and HMGB1 levels, as well as the intima-media thickness (IMT) were increased in hypertensive patients; these effects were reversed following the administration of angiotensin receptor blockers. Based on these observations, we conclude that the downregulation of miR-181b-5p leads to the elevation of HMGB1 levels in hypertensive patients, which accounts, at least partially, for VSMCs phenotypic transformation and vascular remodeling. Our findings also highlight that the plasma levels of miR-181b-5p and HMGB1 may serve as novel biomarkers for vascular remodeling in the hypertensive patients.
\end{abstract}

Key words: HMGB1, hypertension, phenotypic transformation, miR-181b-5p

Hypertension is a common disease and a wide-spread public-health challenge. Over a third of people worldwide have been diagnosed with hypertension [1]. In China, hypertension is considered as a major chronic noninfectious disease and the most important risk factor for cardiovascular diseases. The prevalence of hypertension is $>30 \%$ and the costs represent approximately $6.61 \%$ of the total healthcare budget [2-4]. Extensive efforts have been made to improve the treatment rate for hypertension; however, only approximately $20 \%$ of patients received the treatment [4]. Thus, more studies examining the pathogenesis, effective diagnosis, and low-cost treatments of hypertension remain necessary.

*Correspondence should be addressed to: Dr. Tian-Lun Yang, Xiangya Hospital, Central South University. Email: tianluny@126.com or Dr. Jun Peng, Xianya School of Pharmaceutical Sciences, Central South University, China, Email: Junpeng@csu.edu.cn.

Copyright: () $2018 \mathrm{Li} \mathrm{FJ}$ et al. This is an open-access article distributed under the terms of the Creative Commons Attribution License, which permits unrestricted use, distribution, and reproduction in any medium, provided the original author and source are credited. 
Several mechanisms are responsible for the development of hypertension including excessive vasoconstriction and/or deficient vasodilatation [5]. The remodeling of large and small arteries contributes to the development and complications of hypertension. It is well known that the renin-angiotensin system (RAS) plays a critical role in the development of hypertension and endorgan damage. Angiotensin II (Ang II) is known as a potent vasoconstrictor regulator that elevates blood pressure via activation of the mitogen activated protein kinase (MAPK) signaling pathway. Therefore, angiotensin-converting enzyme inhibitors and angiotensin receptor blockers are used to reduce blood pressure [6-8].

Vascular smooth muscle cells (VSMCs) are a dominant constituent of arteries and a critical determinant of vascular diseases [9]. VSMCs may undergo phenotype alternations between a differentiated phenotype (contractile phenotype) and a dedifferentiated phenotype (synthetic phenotype) in response to different stimuli. The contractile phenotype is characterized by high expression of contractile genes with a low rate of proliferation and migration. Conversely, synthetic VSMCs express low levels of contractile genes with a high rate of proliferation and migration $[10,11]$. Thus, VSMCs phenotypic transformation from contraction to synthesis is widely accepted as the pivotal process in vascular remodeling during hypertension[12].However, the mechanisms responsible for VSMCs phenotypic transformation in hypertension are not fully understood.

Previous studies have demonstrated that low-grade inflammation is involved in the development of hypertension in humans and experimental models. Ang II has been shown to induce inflammatory reactions and Ang II-induced VSMCs phenotypic transformation contributes to the development of hypertension [13-17], suggesting a possible link between Ang II-induced VSMCs phenotypic transformation and inflammation. Recently, high mobility group box-1 (HMGB1) has been proposed as a potent pro-inflammatory cytokine that participates in the development of cardiovascular diseases [18-20]. For example, HMGB1 levels are elevated in atherosclerotic plaques, promoting VSMCs migration and proliferation [5, 21, 22]. Based on these reports, it is reasonable to speculate that HMGB1 plays a role in the mediation of Ang II- induced VSMCs phenotypic transformation in hypertension.

MicroRNAs (miRNAs), small non-coding RNAs, are well-known as negative regulators of the mRNA expression of target genes [6, 23]. Certain miRNAs have been shown to regulate VSMCs functions such as migration and proliferation [24, 25]. Abnormal expression of miRNAs is involved in a variety of human diseases including coronary heart diseases, hypertension, and vascular aneurysms $[26,27]$. However, it is unknown whether certain miRNAs are involved in the Ang IIinduced VSMCs phenotypic transformation in hypertension.

The goals of this study were as follows: 1) to investigate the roles of HMGB1 in Ang II-induced VSMCs phenotypic transformation, migration, and proliferation; 2) to identify miRNAs involved in the regulation of HMGB1 expression; and 3) to determine whether the miRNAs/HMGB1pathway is involved in vascular remodeling in hypertension patients with high plasma Ang II levels.

\section{MATERIALS AND METHODS}

\section{In vitro study}

\section{Protocol for cell experiments}

Human aortic vascular smooth muscle cells (HAVSMCs) were obtained from the Central South University Cell Center and cultured in high glucose Dulbecco's Modified Eagle's Medium (DMEM, Biological Industries, israel) containing $10 \%$ fetal bovine serum (FBS, Biological Industries, israel). Cells were incubated at $37{ }^{\circ} \mathrm{C}$ in a humidified atmosphere of $95 \%$ air and $5 \% \mathrm{CO}_{2}$ and subcultured every 3 or 4 days with trypsin $(0.25 \%)$ added EDTA $(0.01 \%)$.

To explore the role of HMGB1 in Ang II-induced phenotypic transformation of HAVSMCs, HAVSMCs were randomly divided into three groups: control, without any treatment; Ang II, cells incubated with Ang II $(1 \mu \mathrm{M})$ for $48 \mathrm{~h}$ to induce phenotypic transformation; and Ang II + losartan, cells pretreated with losartan $(10 \mu \mathrm{M})$ for $1 \mathrm{~h}$ before prior to Ang II treatment.

To confirm the function of HMGB1 in mediating the Ang II-induced phenotypic transformation of HAVSMCs, HAVSMCs were randomly divided into four groups: the control group, the Ang II group, the HMGB1 siRNA group (cells transfected with $50 \mathrm{nM}$ of the HMGB1siRNA for $24 \mathrm{~h}$ prior to Ang II treatment), and the siRNA negative control (NC) group (cells transfected with 50nM of the siRNA NC for $24 \mathrm{~h}$ prior to Ang II treatment).

To determine whether miR-181b-5p is an upstream regulator of HMGB1 in Ang II-treated HAVSMCs, HAVSMCs were randomly divided into four groups: the control group, the Ang II group, the miR-181b-5p mimic group (cells transfected with $50 \mathrm{nM}$ of the miR-181b-5p mimic for $24 \mathrm{~h}$ prior to Ang II treatment), and the mimic $\mathrm{NC}$ group (cells were transfected with $50 \mathrm{nM}$ of the mimic $\mathrm{NC}$ for $24 \mathrm{~h}$ prior to Ang II treatment).

At the end of the experiments, cells were collected for analysis of parameters relevant to phenotypic transformation and molecular studies (miRNA, mRNA, or protein expression). 
Table 1. The primary antibodies for Western Blot.

\begin{tabular}{cccl}
\hline $\begin{array}{c}\text { Product } \\
\text { Number }\end{array}$ & $\begin{array}{c}\text { Protein } \\
\text { Name }\end{array}$ & $\begin{array}{c}\text { Dilution } \\
\text { ratio }\end{array}$ & Manufacturers \\
\hline ab79823 & HMGB1 & $1: 10000$ & abcam, USA \\
ab32575 & $\alpha-S M A$ & $1: 1000$ & abcam, USA \\
ab14106 & SM22 $\alpha$ & $1: 1000$ & abcam, USA \\
ab91655 & OPN & $1: 5000$ & abcam, USA \\
A531 & GAPDH & $1: 10000$ & Bioworld, USA \\
\hline
\end{tabular}

\section{Cell transfection experiments}

The human HMGB1 small interference RNA (siRNA), miR-181b-5p mimic, NC siRNA, NC mimic, and transfection reagent were all purchased from RiboBio (Guangzhou, China). qPCR assays were used to determine the transfection efficiency. HAVSMCs grown to $50 \%$ to $60 \%$ confluence were starved using high glucose DMEM with $0.5 \%$ FBS for $24 \mathrm{~h}$ and then transfected using the riboFECTTM transfection system for $24 \mathrm{~h}$. Prior to the above-mentioned experiments, all of the miRNA mimics and HMGB1 siRNAs used in this study were confirmed to work as expected. All transfections followed the manufacturer's instructions.

\section{Cell migration assay}

HAVSMCs migration was evaluated using woundhealing and Transwell migration assays. HAVSMCs were seeded in 6-well plates and the monolayers were scratched using sterile pipette tips. The cells were washed with PBS and then fresh DMEM with $0.5 \%$ FBS was added. The wound gaps were recorded using bright-field microscopy at $0 \mathrm{~h}$ and $48 \mathrm{~h}$ and measured with the Image-pro plus 6.0 software. The migration distance was analyzed by initial wound distance subtracting the remaining distance.

For the Transwell migration assays, HAVSMCs were seeded at a density of $1 \times 10^{5}$ cells/well into the upper chambers, containing a filter membrane $(8-\mu \mathrm{m}$ pore size $)$ of 24-well Transwell plates (Corning Inc., New York, USA). The lower chambers were then filled with $0.5 \%$ FBS medium containing $20 \mathrm{ng} / \mathrm{ml}$ platelet-derived growth factor-BB (PDGF-BB; PeproTech, USA) and the plate was further incubated for $12 \mathrm{~h}$. Migrated cells on the bottom of the filter were fixed with $4 \%$ triformol then stained using a crystal violet solution (Beyotime, Nanjing, China) and imaged by bright-field microscopy.

\section{Cell proliferation assay}

VSMCs proliferation was evaluated using the Edu incorporation assay according to the manufacturer's instructions (RiboBio, R11053.2, Guangzhou, China). Briefly, HAVSMCs were seeded at a density of $6 \times 10^{3}$ cells/well in 96-well plates and incubated with Edu for $2 \mathrm{~h}$, then fixed, stained, and imaged by fluorescence microscopy. The Edu-positive cells were counted and normalized to the total number of DAPI-stained cells [28].

\section{Cell viability}

Cell viability was evaluated using Cell Counting Kit-8 (CCK-8; Bimake, USA) according to the manufacturer's protocol. Briefly, HAVSMCs were seeded at a density $6 \times 10^{3}$ cells/well in 96-well plates and incubated with the CCK-8 solution for $1 \mathrm{~h}$. The absorbance of the samples was measured at $450 \mathrm{~nm}$ using a microplate reader. Blank wells with $0.5 \%$ FBS were used at the blank control group.

\section{Protein extraction and western blot analyses}

Total proteins were extracted from HAVSMCs using RIPA lysis buffer (Beyotime, Nanjing, China). The BCA Protein Assay Kit (Beyotime, Nanjing, China) was used to determine the total protein concentrations. A standardized amount of total protein (20-40 $\mu \mathrm{g})$ from each sample was separated using $12 \%$ sodium dodecyl sulfate polyacrylamide gel electrophoresis. Following separation, proteins were transferred onto polyvinylidenefluoride membranes (Millipore, USA) and blocked with 5\% nonfat milk at room temperature for $1 \mathrm{~h}$. The membranes were washed with TBST three times then incubated with primary antibodies at $4{ }^{\circ} \mathrm{C}$ overnight. After three washes, the membranes were incubated with peroxidaseconjugated affinipure goat anti-rabbit igg secondary antibody (ZB-5301, ZSGB-BIO, Beijing, China) at room temperature for $1 \mathrm{~h}$. The chemiluminescence signals were detected using the BeyoECL Star Kit (Beyotime, Nanjing, China). Densitometric analysis was conducted using Image J 1.43 software (National Institutes of Health New York City, NY, USA). GAPDH was used as the internal control. All primary antibody manufacturers and concentrations used in these experiments are detailed in Table 1.

\section{RNA extraction and quantitative real-time polymerase chain reaction}

Total RNA was extracted from the HAVSMCs using the Trizol reagent (Invitrogen, Waltham, MA, USA). RNAs enriched for small size were extracted from $200 \mu \mathrm{l}$ of plasma using the EasyPure ${ }^{\circledR}$ miRNA Kit (ER601, TransGen Biotech, Beijing, China) according to the manufacturer's protocol; $5 \mu 1$ of $5 \mathrm{nM}$ Syn-cel-miR-39 miScript miRNA Mimic (RiboBio) were added to each 
sample prior to the addition of acid-phenol:chloroform [29].

Table 2. Human Primer sequences for quantitative real-time PCR.

\begin{tabular}{ll}
\hline Gene & Forward (F) and reverse (R) \\
\hline GAPDH & F:5'-TGATGACATCAAGAAGGTGGTGAAG \\
& R:3'-TCCTTGGAGGCCATGTGGGCCAT \\
$\alpha$-SMA & F:5'-AGCGTGGCTACCCTTCGTGAC \\
& R:3'-GCTCGTTGCCGATGGTGATGAC \\
SM22 $\alpha$ & F:5'-GAATGGCGTGATTCTGAGC \\
& R:3'-CTCCATCTGCTTGAAGAC \\
OPN & F:5'- TGAGTCTGGAAATAACTAATGTGTTTGA \\
& R:3'-GAACATAGACATAACCCTGAAGCTTTT \\
HMGB1 & F:5'-CACTGGGCGACTCTGTGCCTCG \\
& R:3'-CGGGCCTGTCCGCTTTGCCA \\
HMGB1_mut & F:5'-AGACCTGACTTACATCCCCAAAAGCGTA \\
& GCT \\
& R:3'-TTTGGGGATGTAAGTCAGGTCTTCTTTAA \\
& TGT \\
HMGB1- & F:5'-GCGGCTCGAGATCAATCTACTCAAAGCAT \\
3'UTR & R:3'-AATGCGGCCGCAACTCCTAAGCAGATA \\
& AAC \\
\hline
\end{tabular}

RNA (0.2-0.5 $\mu \mathrm{g})$ was used as template for reverse transcription using the PrimeScript reverse transcription reagent Kit (DRR037S; TaKaRa, Dalian, China). The change in RNA expression levels was determined using SYBR ${ }^{\circledR P r e m i x}$ Ex Taq ${ }^{\mathrm{TM}}$ (DRR42OA, TaKaRa) with the ABI 9700 software (Applied Biosystems, Waltham, MA, USA) and the following conditions: denaturation at $95^{\circ} \mathrm{C}$ for $10 \mathrm{~min}$ and 45 amplification cycles (denaturation at $95^{\circ} \mathrm{C}$ for $15 \mathrm{~s}$, annealing and extension at $60^{\circ} \mathrm{C}$ for $1 \mathrm{~min}$ ). Data analysis was performed using the comparative $\mathrm{Ct}$ method. Absolute quantitation of miRNA concentration in plasma samples was also directly determined using the same SYBR ${ }^{\circledR}$ Premix Ex Taq ${ }^{\mathrm{TM}}$ miRNA assays. The target gene mRNA results were normalized to GAPDH mRNA. For the measurement of miRNA expression, U6 or celmiR-39 was used as an internal control (for HAVSMCs) or external control (for plasma) to normalize miRNA expression in different groups. All miRNA primers, including cel-miR-39, were purchased from RiboBio and other target genes primers were purchased from Sangon Biotech (Shanghai, China). The primer sequences are presented in Table 2 and Table 3.

\section{Luciferase activity assay}

The 3'-UTR of the HMGB1 mRNA fragment containing the putative miR-181b-5p-binding sequence was amplified by PCR. The primers for PCR amplification are presented in Table 2. The PCR product was inserted at the NotI and $\mathrm{XhoI}$ sites and the resulting construct was named WT+HMGB1-3'UTR. The HMGB1 mutant was constructed similarly to the HMGB1 wild type, except that the wild type target sequence "GAATGTA" was replaced with the mutated sequence "CTTACA"; this construct was named MUT+HMGB1-3'UTR.

The products were cloned into the pmiRREPORTTM luciferase reporter vector and then cotransfected with miR-181b-5p mimic $(50 \mathrm{nM})$ or $\mathrm{NC}$ mimic $(50 \mathrm{nM})$. This mixture was transfected into $293 \mathrm{~T}$ cells according to the instructions provided by the Attractene Transfection Reagent kit (Invitrogen, USA). Following transfection for 24h, the Light Switch Luciferase Assay Reagent (Dual-Glo®Luciferase Assay System, Promega, USA) was added to measure luciferase activity with an illuminometer. These experiments were performed in triplicate.

\section{Clinical Study}

The clinical study protocol was approved by the Hospital Ethics Committee and conformed to the principles outlined in the Declaration of Helsinki; written informed consent was obtained from all subjects.

Table 3. The miRNA sequences and miRBase accession numbers.

\begin{tabular}{lll}
\hline miRNA & \multicolumn{1}{c}{ Sequence } & Number \\
\hline hsa-miR-103a-3p & AGCAGCAUUGUACAGGGCUAUGA & MIMAT0000101 \\
hsa-miR-107 & AGCAGCAUUGUACAGGGCUAUCA & MIMAT0000104 \\
hsa-miR-181b-5p & AACAUUCAUUGCUGUCGGUGGGU & MIMAT0000257 \\
\hline
\end{tabular}

\section{Subjects and protocol}

A total of 182 subjects ( 90 men and 92 women) aged 18 to 78 years were recruited for this study from June 2016 to June 2017 at the Department of Cardiology and the Physical Examination Center of Xiangya Hospital, Central South University, Changsha, China. Patients with concomitant valvular heart disease, congenital heart disease, cardiomyopathy, acute and chronic viral or bacterial infection, asthma, tumors, and connective tissue diseases were excluded. Patients were also excluded at screening if they had a history of severe hypertension $(\geq 180 / 110 \mathrm{mmHg})$ and any secondary hypertension. Additional exclusion criteria included a history of diabetes mellitus; history of cardiovascular disease, including coronary artery disease, myocardial infarction, 
stroke, transient ischemic attack, any revascularization procedure, congestive heart failure, or thermodynamically significant carotid or peripheral arterial disease; systemic hypertension, hypercholesterolemia; sepsis; abnormal liver and renal function or syndrome; electrolyte disturbance; and chromosomal disorders. Patients using any medications likely to affect blood pressure, including non-steroidal anti-inflammatory drugs; glucocorticoids, potent CYP3A4 inhibitors, or potassium supplements were also excluded from the study [30]. Female patients were required to not have been pregnant for 1 year. Detailed health history and demographic data including age, gender, blood pressure, fasting bloodglucose (FBG), triglyceride (TG), total-cholesterol (TC), high-densitylipoprotein-cholesterol (HDL-C), low-density-lipoproteincholesterol(LDL-C), alanine transaminase (ALT), aspartate transaminase (AST), blood urea nitrogen $(\mathrm{BUN})$, uric acid (UA), creatinine (Cr), electrocardiography, echocardiography, and carotid artery ultrasound were collected. Biochemical assessments were performed under fasting conditions in the early morning.

A total of 58 age-matched healthy subjects were recruited as controls. Eligible patients were 18 to 78 years old with stage 1 to 2 hypertension, either untreated or treated with an angiotensin receptor blocker (ARB). The patients without any treatment were defined as the hypertension group $(\mathrm{n}=53)$ and the patients treated solely with ARB were defined as the ARB group $(n=71)$. Essential hypertension was diagnosed according to the American Joint National Committee 2014 EvidenceBased Guideline for the Management of High Blood Pressure in Adults.

\section{Carotid Ultrasound Studies}

Carotid ultrasound examination was performed using a USA HP SONOS5500 Color Doppler Ultrasonic Diagnosis Apparatus equipped with a 7.5-MHz transducer frequency. The electrocardiogram $\mathrm{R}$ wave vertex measured the posterior wall of the carotid artery lumenintima interface (Lumen-Intima, LI) and the media membrane-adventitia membrane interface (MediaAdventitia, MA). The maximum range of LI and MA constitutes the carotid intima media thickness (IMT). Continuous measurement was performed for three cardiac cycles and the mean value served as the IMT value, which was expressed in $\mathrm{mm}$.

\section{Blood sampling}

Peripheral blood $(10 \mathrm{ml})$ was obtained from the elbow vein of all subjects and collected in tubes with EDTA. The plasma was harvested from the upper layer following centrifugation at $3000 \mathrm{rpm}$ for $10 \mathrm{~min}$ and stored at $-80^{\circ} \mathrm{C}$ for subsequent measurements including HMGB1, Ang II, and miRNAs.

\section{Measurements of the HMGB1 and Ang II levels}

The levels of HMGB1 or Ang II in culture medium or plasma were measured using a commercially available ELISA Kit (Uscn Life Science, Wuhan, China). Following the manufacturer's instructions, the absorbance was measured at $450 \mathrm{~nm}$ and the levels of HMGB1 or Ang II were calculated according to a standard curve.

\section{Statistical analysis}

All data were expressed as mean \pm SEM. Patient characteristics were compared using the Student's $t$ test or the chi-square test. Correlations were tested by calculating the Pearson's correlation coefficient. Comparisons of multiple groups were performed with one-way analysis of variance (ANOVA). Differences were considered statistically significant at $P<0.05$.

\section{RESULTS}

\section{Up-regulation of HMGB1 in Ang II-induced phenotypic transformation of HAVSMCs}

To explore the role of HMGB1 in hypertension, Ang II was used to induce the phenotypic transformation of HAVSMCs. Following incubation with Ang II $(1 \mu \mathrm{M})$ for 48h, HAVSMCs exhibited phenotypic transformation from a contractile to a synthetic type, as evidenced by down-regulation of contractile proteins $\alpha$-smooth muscle actin ( $\alpha$-SMA) and smooth muscle $22 \alpha$ (SM22 $\alpha$ ) (Fig. 1 A, B, E, F, G) and up-regulation of the synthetic protein osteopontin (OPN) (Fig. 1C, E, H); these phenomena were abolished in the presence of losartan (an AT1 receptor blocker), suggesting that Ang II successfully induced the phenotypic transformation of HAVSMCs. Compared to the control group, HMGB1 mRNA and protein expression was up-regulated in Ang II-treated HAVSMCs, which was suppressed in the presence of losartan (Fig. 1D, E, I, J).

\section{Knockdown of HMGBI suppressed Ang II-induced HAVSMC phenotype transformation}

To confirm the relationship between HMGB1 and Ang IIinduced HAVSMC phenotype transformation, siRNA was used to knockdown HMGB 1 (Fig. 2A and B). Similar to the results with losartan, knockdown of HMGB1 attenuated Ang II-induced phenotypic transformation of HAVSMCs, as shown by the recovery of $\alpha$-SMA and SM22 $\alpha$ levels (Fig. 2C and D) as well as OPN levels (Fig. 
2E). The negative control (NC) HMGB1 siRNA did not demonstrate similar effects.
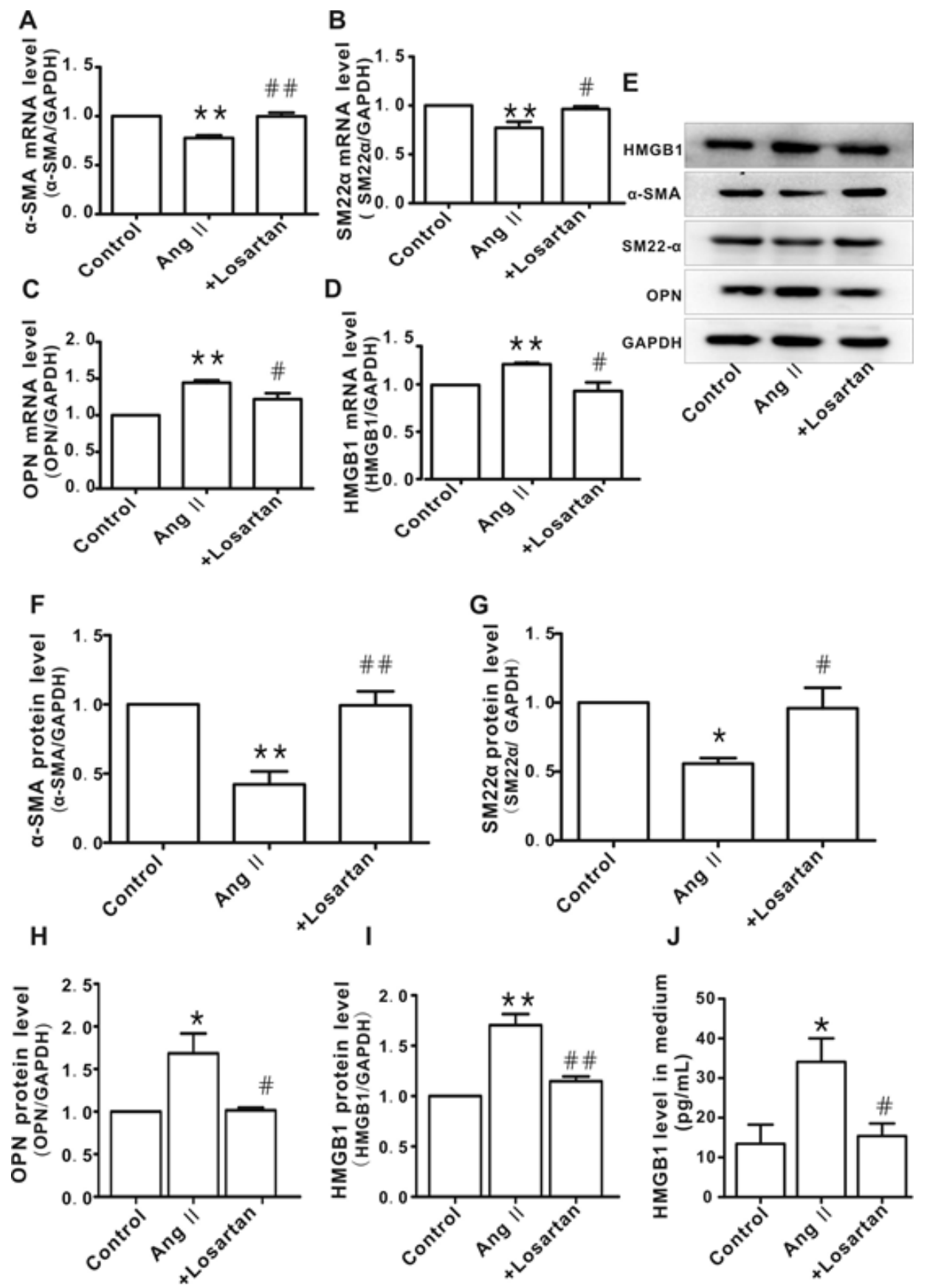

Figure 1. Up-regulation of high-mobility group box 1 (HMGB1) in angiotensin (Ang) IIinduced human aortic vascular smooth muscle cell (HAVSMC) phenotype transformation. A-D) mRNA levels of $\alpha$-smooth muscle actin ( $\alpha$-SMA), smooth muscle $22 \alpha$ (SM22 $\alpha$ ), osteopontin (OPN), and HMGB1 in HAVSMCs, respectively. E) representative images of western blot analysis for HMGB1, $\alpha$-SMA, SM22 $\alpha$, OPN, and GAPDH. F-I) Protein levels of $\alpha$-SMA, SM22 $\alpha$, OPN, and HMGB1, respectively. Arbitrary optical density units of the target proteins were normalized to GAPDH and expressed as fold change. J. Protein levels of HMGB1 in culture medium $* P<0.05$, ${ }^{* *} P<0.01$ vs. control group; ${ }^{\#} P<0.05,{ }^{\#} P<0.01$ vs. Ang II group. At least three independent experiments were performed for each group. 
A

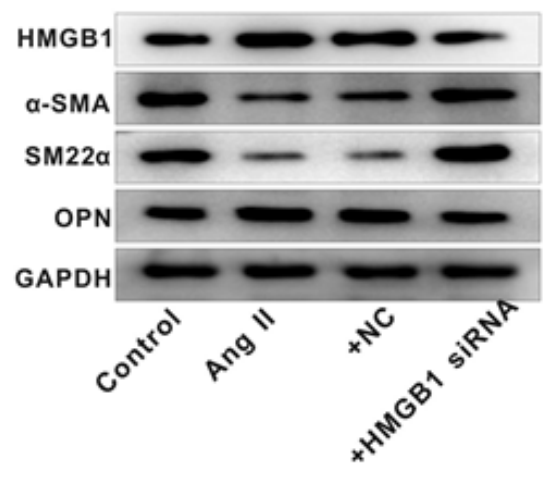

C

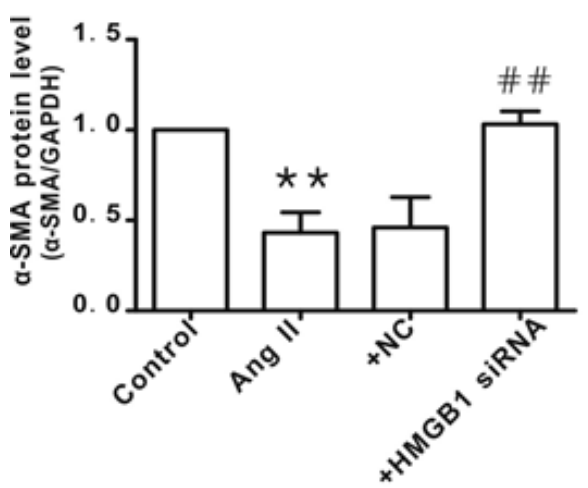

E

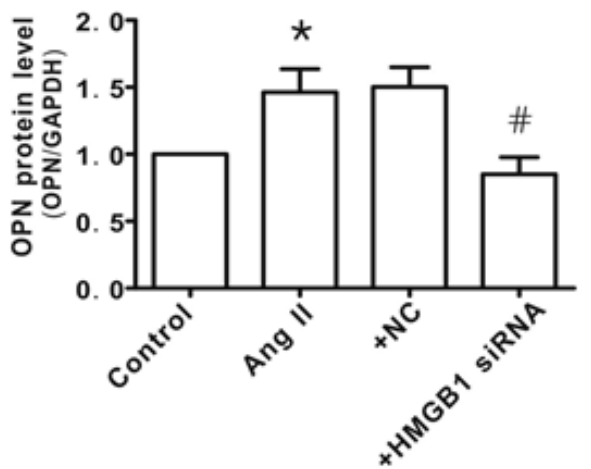

B

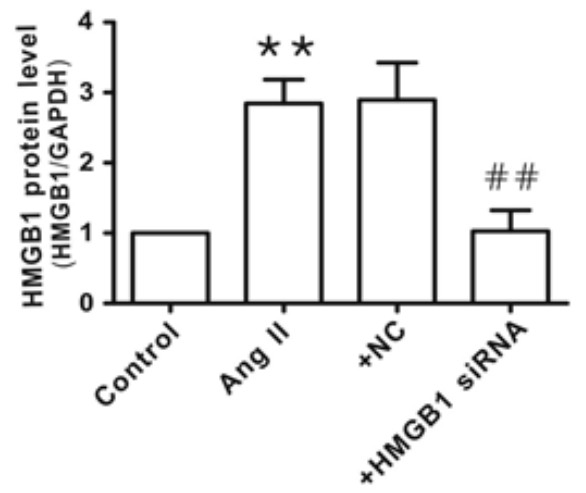

D

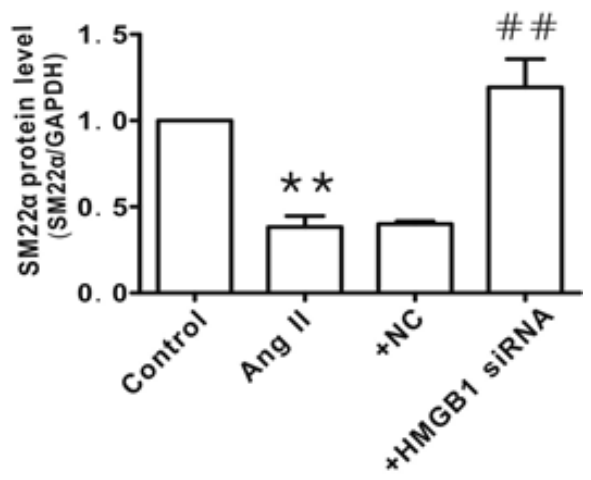

Figure 2. HMGB1 silencing inhibited Ang II-induced HAVSMC phenotype transformation. A) Representative images of western blot analysis for HMGB1, $\alpha$-SMA, SM22 $\alpha$, OPN, and GAPDH. B-E) Protein levels of HMGB1, $\alpha$-SMA, SM22 $\alpha$, and OPN, respectively. Arbitrary optical density units of the target proteins were normalized to GAPDH and expressed as fold change. ${ }^{*} P<0.05,{ }^{* *} P<0.01$ vs. control group; ${ }^{\#} P<0.05$, ${ }^{\# \#} P<0.01$ vs. the Ang II group. At least three independent experiments were performed for each group.

\section{Knockdown of HMGBI blocked Ang II-induced HAVSMC migration and proliferation}

To verify the function of HMGB1 in the mediation of Ang II-induced HAVSMC phenotype transformation, cellular migration and proliferation were measured. As shown in Fig. 3A-F, silencing of HMGB1 blocked Ang II-induced
HAVSMC migration, as determined by the scratchwound healing assay (Fig. 3A and D) and Transwell chamber assay (Fig. 3B and E). Silencing of HMGB1 also inhibited HAVSMC proliferation and cells viability, as revealed by the Edu (Fig. 3C and F) and CCK8 assay (Fig. $3 G)$. The NC HMGB1 siRNA did not display such effects. 
A
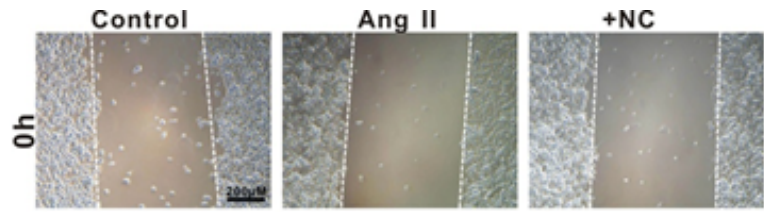

+HMGB1 SIRNA
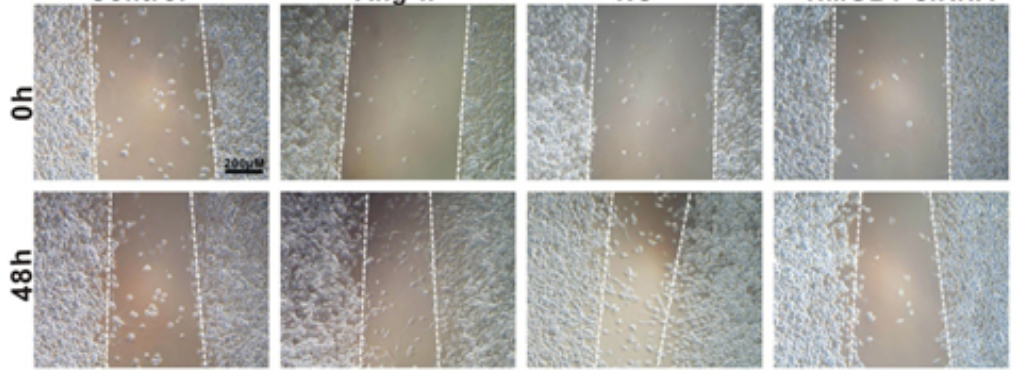

B
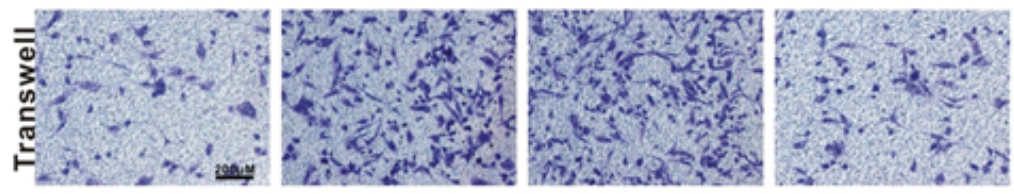

C
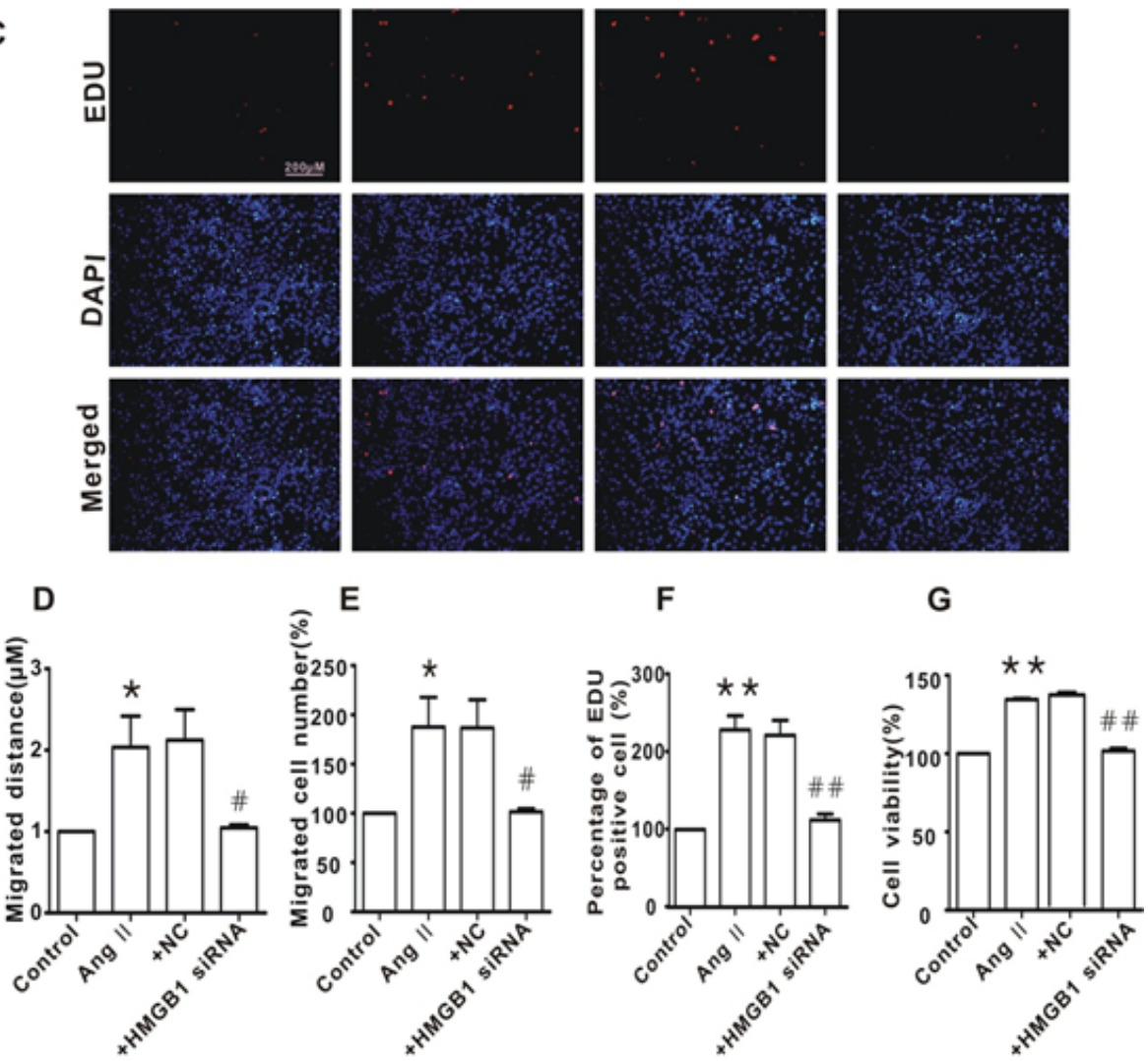

Figure 3. HMGB1 silencing inhibited HAVSMC migration, proliferation, and cell viability. A) Representative images of scratch-wound healing assay $(100 \times)$. B) Representative images of Transwell assay $(100 \times)$. C) Representative images of Edu assay $(100 \times)$. D) HAVSMC migration distance in each group. E. The number of migrated HAVSMCs in each group. F) The percentage of Edu-positive cells in each group. G. Cell viability in each group. ${ }^{*} P<0.05,{ }^{* *} P<0.01$ vs. the control group; ${ }^{\#} P<0.05,{ }^{\#} P<0.01$ vs. the Ang II group. At least three independent experiments were performed for each group.

\section{Identification of miR-181b-5p as an upstream regulator of HMGB1 in Ang II-treated HAVSMCs}

As shown in Fig. 4A and $4 \mathrm{~B}$, there are three putative miRNA binding sites in the HMGB1 3'-UTR (as determined by computational analysis using target scan software), which are highly conserved among humans (Fig. 4C). However, only miR-181b-5p was significantly decreased in Ang II-treated HAVSMCs and this decrease was attenuated in the presence of losartan (Fig. 4D, $P<0.01)$. 
A Human miR-103a-3p/107/181b-5p

\begin{tabular}{|c|c|c|c|c|c|c|c|c|}
\hline \multirow[b]{2}{*}{ Target gene } & \multirow[b]{2}{*}{$\begin{array}{l}\text { Representative } \\
\text { transcript }\end{array}$} & \multirow[b]{2}{*}{ Gene name } & \multirow{2}{*}{$\begin{array}{c}\text { Number of } \\
3 \text { P-seq } \\
\text { tags } \\
\text { supporting } \\
\text { UTR }+5\end{array}$} & \multirow[b]{2}{*}{$\begin{array}{l}\text { Link to } \\
\text { sites in } \\
\text { UTRs }\end{array}$} & \multicolumn{4}{|c|}{ Conserved sites } \\
\hline & & & & & tota & | $8 \mathrm{mer}$ & $\begin{array}{c}7 \mathrm{mer}- \\
\mathrm{m} 8\end{array}$ & $\begin{array}{r}7 \mathrm{me} \\
\text { A1 }\end{array}$ \\
\hline FURIN & ENST00000268171.3 & furin (paired basic amino acid cleaving enzyme) & 2306 & Sites in UTR & 1 & 0 & 1 & 0 \\
\hline NMT2 & ENST00000378165.4 & $\mathrm{N}$-myristoyitransferase 2 & 318 & Sites in UTR & 1 & 0 & 0 & 1 \\
\hline HMGB1 & ENSTO0000399489.1 & high mobility group box 1 & 800 & Sites in UTR & 1 & 0 & 0 & 1 \\
\hline BAZ2A & ENST00000379441.3 & bromodomain adjacent to zinc finger domain, $2 \mathrm{~A}$ & 48 & Sites in UTR & 2 & 0 & 2 & 0 \\
\hline
\end{tabular}

\section{B Human HMGB1 3'UTR}

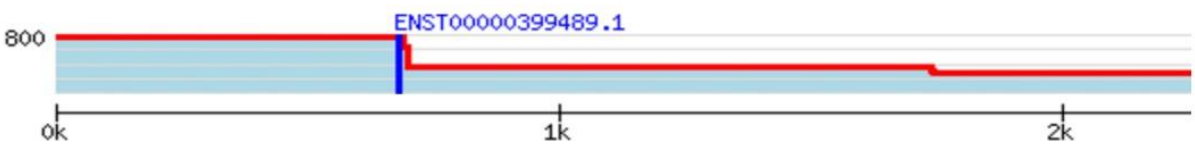

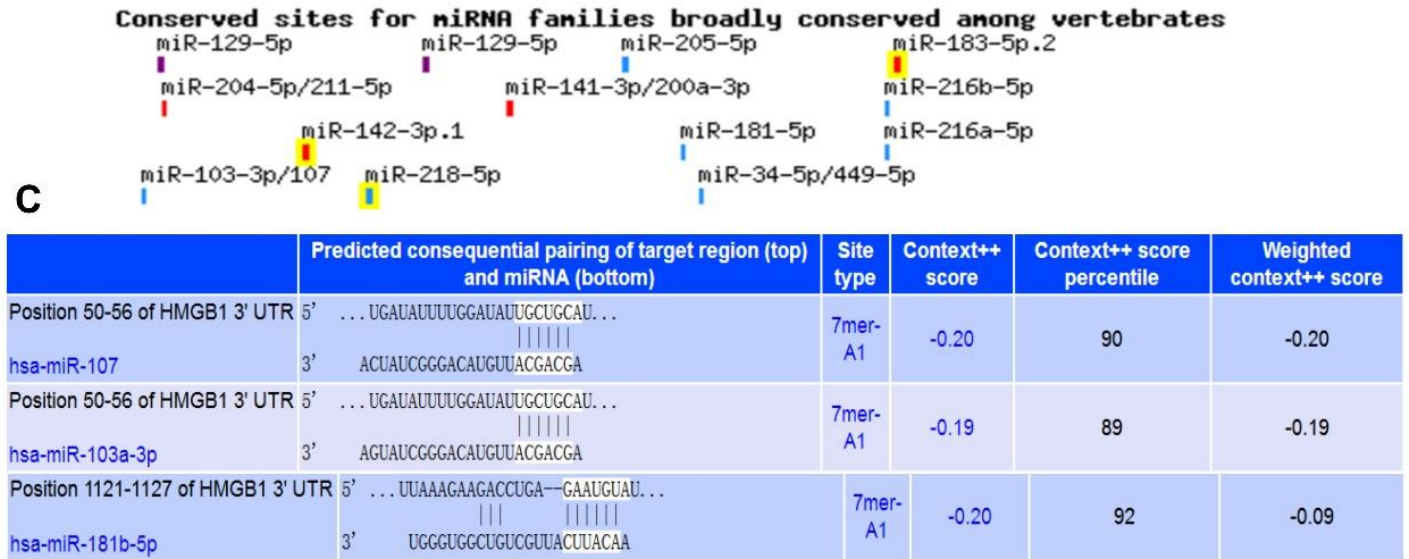

D

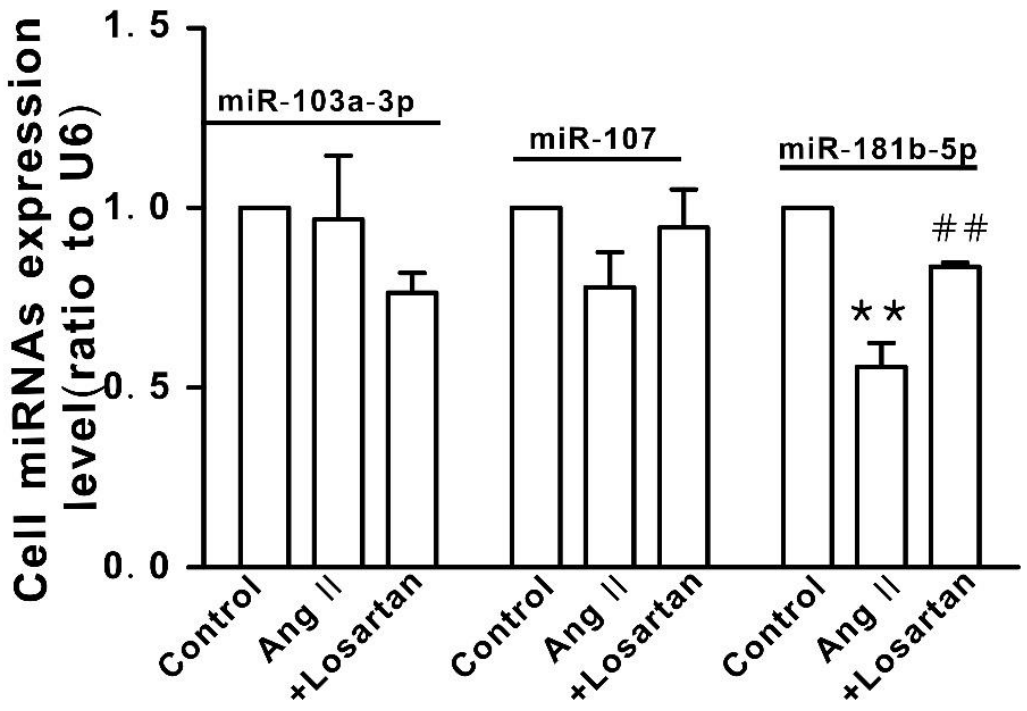

Figure 4. Putative binding sites of miR-181b-5p in the HMGB1 3 '-UTR. A and B) The three putative miRNA binding sites in the HMGB1 3'-UTR predicted by target scan analysis. C) Conserved putative target sites of HMGB1 in humans. D) Expression of the putative miRNAs in HAVSMCs. ${ }^{* *} P<0.01$ vs. the control group; ${ }^{\# \#} P<0.01$ vs. the Ang II group. At least three independent experiments were performed for each group. 


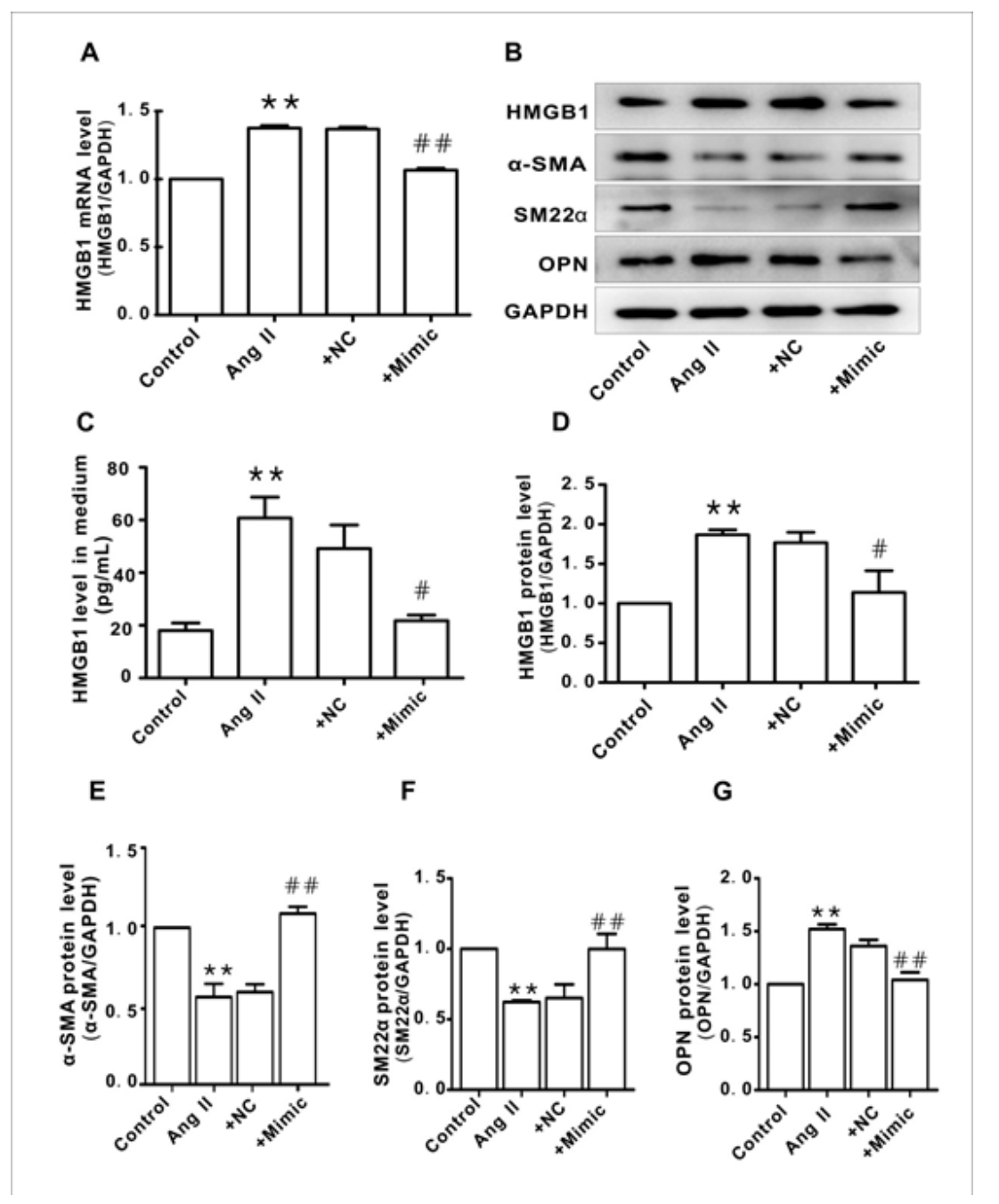

Figure 5. Overexpression miR-181b-5p reversed Ang II-induced HAVSMC phenotype transformation. A. HMGB1 mRNA expression in each group. B. Representative images of western blot analysis for HMGB1, $\alpha$-SMA, SM22 $\alpha$, OPN, and GAPDH. C. Protein levels of HMGB1 in culture medium. D-G. Protein levels of HMGB1, $\alpha$-SMA, SM22 $\alpha$, and OPN, respectively. Arbitrary optical density units of the target proteins were normalized to GAPDH and expressed as fold change. ${ }^{*} P<0.05$, ${ }^{*} * P<0.01$ vs. the control group; ${ }^{\#} P$ $<0.05,{ }^{\# \#} P<0.01$ vs. the Ang II group. At least three independent experiments were performed for each group.

To confirm the link between miR-181b-5p and HMGB1, miR mimics were applied. As shown in Fig. 5, the miR-181b-5p mimic markedly suppressed HMGB1 expression (Fig. 5A-D) and reversed the phenotype transformation in Ang II-treated HAVSMCs, as evidenced by the recovery of $\alpha$-SMA and SM22 $\alpha$ levels (Fig. 5E and F) as well as OPN levels (Fig. 5G). Similarly, the miR-181b-5p mimic also blocked Ang II-induced HAVSMC migration (Fig. 6A, B, D, E) and proliferation (Fig. 6C, F, G). The NC of the miR-181b-5p mimic did not exert any obvious effect on HMGB1 expression and phenotypic transformation.

To confirm that HMGB1 is a direct target of miR$181 b-5 p$, dual luciferase reporter vectors containing wildtype (WT) or mutant HMGB1 3'-UTR were constructed (Fig. 7A and B). The results of the luciferase assay indicated that miR-181b-5p inhibited luciferase activity in the HMGB1 WT 3'-UTR group; however, the inhibitory effects were abolished when the HMGB1 3'-UTR binding site was mutated (Fig. 7C).

\section{Clinical characteristics}

This study specifically focused on essential hypertension. The clinical study included 182 subjects in total; these were divided into three groups based on clinical circumstances and research objectives as follows: healthy controls served as the normal group $(n=58)$, patients not receiving any anti-hypertension medication served as the hypertension group $(n=53)$, and patients treated solely with an angiotensin receptor blocker (ARB) served as the ARB group $(\mathrm{n}=71)$.

There were no significant differences in gender, age, FBG, TG, TC, HDL-C, LDL-C, ALT, AST, BUN, UA and $\mathrm{Cr}$ between the three groups, except for the systolic blood pressure (SBP) and diastolic blood pressure (DBP). Compared to the control group, the SBP and DBP were obviously elevated in the hypertensive patients; these values were significantly decreased subsequent to ARB (such as losartan, valsartan, or Irbesartan) administration for over three months (Table 4, Fig. 8A and B). 
A
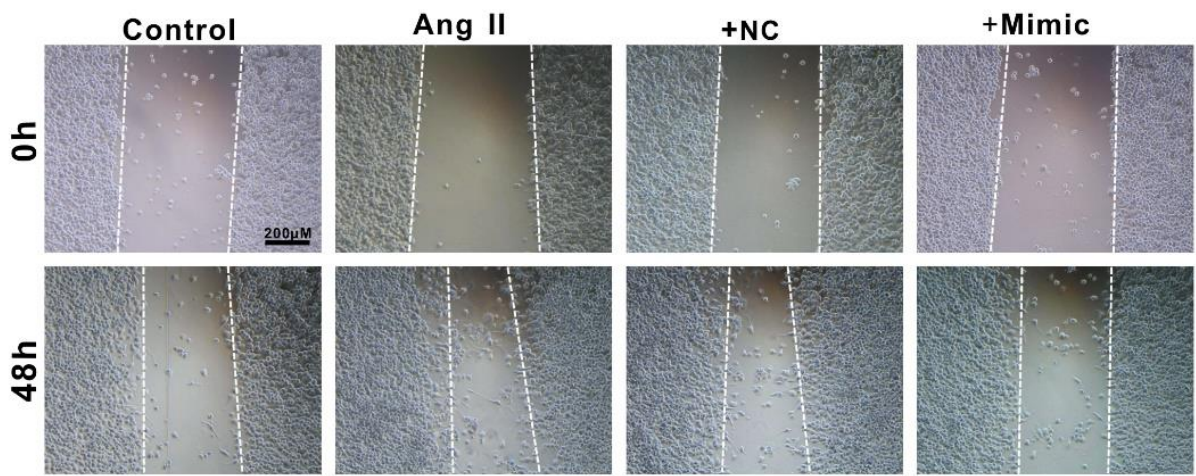

B
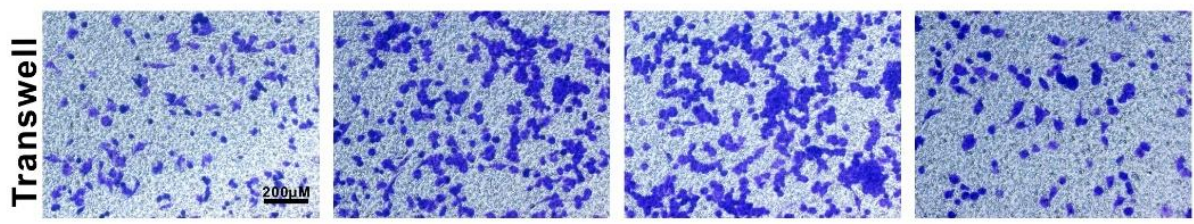

C
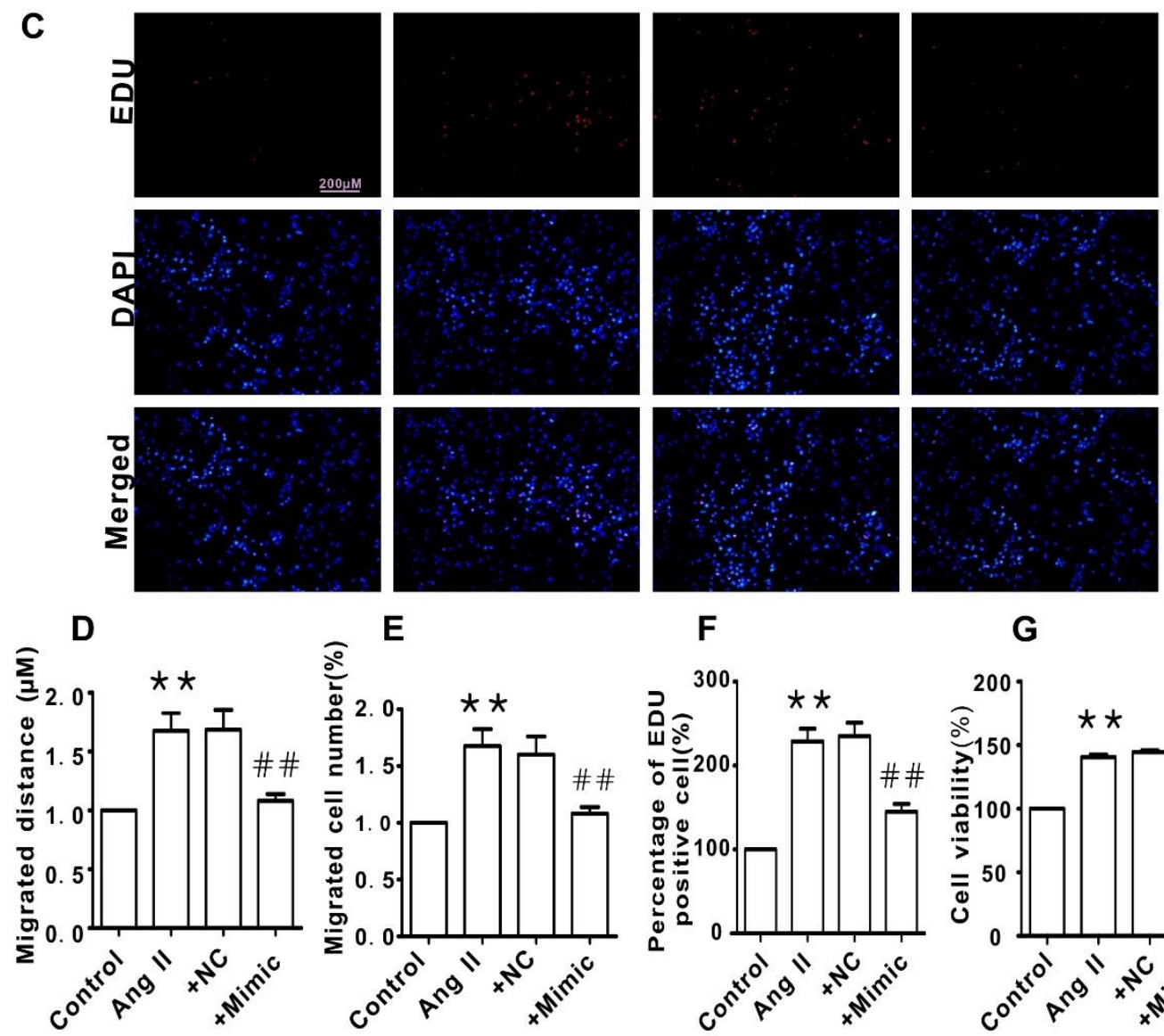

$\mathbf{F}$

G
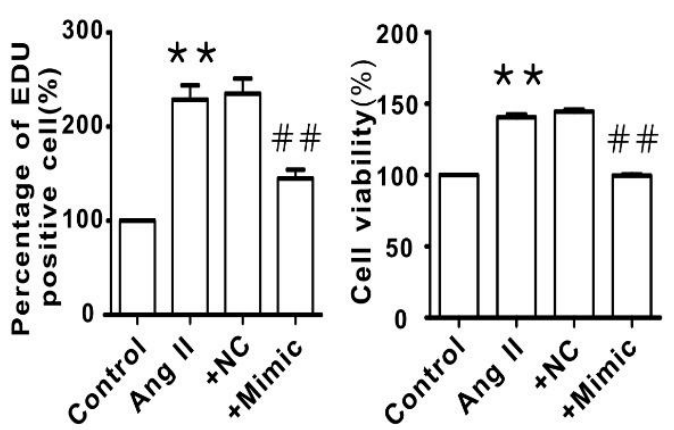

Figure 6. Overexpression miR-181b-5p inhibited HAVSMC migration, proliferation, and cell viability. A) Representative images of scratch-wound healing assay (100×). B) Representative images of Transwell assay (100x). C. Representative images of Edu assay (100x). D) HAVSMC migration distance in each group. E) The number of migrated HAVSMCs in each group. F) The percentage of Edu-positive cells in each group. G) Cell viability in each group. ${ }^{*} P<0.05,{ }^{*} P<0.01$ vs. the control group; ${ }^{\#} P<0.05$, ${ }^{\# \#} P<0.01$ vs. the Ang II group. At least three independent experiments were performed for each group. 
A

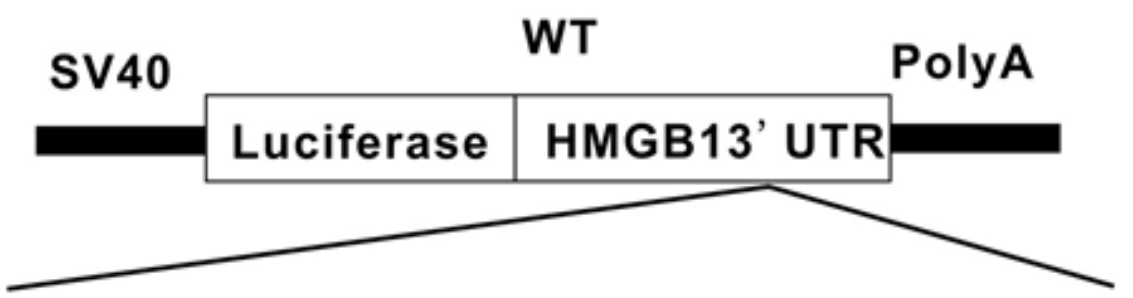

GAGCTCATCAATCTACTCAAAGCATGGGATTATTAGAATCAAACA......TGCAGTTCCCACATTAAAGAAGACC IGAGAATGTATCCCCAAAAGCGTGAGCTTAAAATACAAGACTGCCATATTAAATTTTTTGTTGACATTAGTC TCAGTGAAGACTATGAAAATGCTGGCTATAGATGTCTTITCCCATTTATCTAAATATGGACTGCTCAGGAAACG AGACTTTCATTACAAGTA.......TTTGCATCTCAGTATGAATTATTCAATTTATTTGAATGATTTTTCTTTACAAAA CAAACTCATTCATTAGTCATGTITATCTGCTTAGGAGTTTACGCGT

B

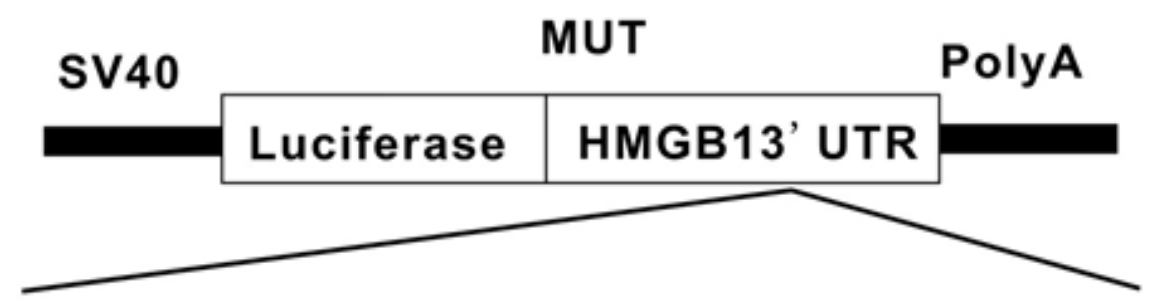

GAGCTCATCAATCTACTCAAAGCATGGGATTATTAGAATCAAACA......TGCAGTTCCCACATTAAAGAAGACC IGACTTACATCCCCAAAAGCGTGAGCTTAAAATACAAGACTGCCATATTAAATTTTTTGTTGACATTAGTCTC AGTGAAGACTATGAAAATGCTGGCTATAGATGTCTTTTCCCATTTATCTAAATATGGACTGCTCAGGAAACGA GACTTTCATTACAAGTA.......TTTGCATCTCAGTATGAATTATTCAATTTATTTGAATGATTTTTCTTTACAAAAC AAACTCATTCATTAGTCATGTTTATCTGCTTAGGAGTTTACGCGT

C

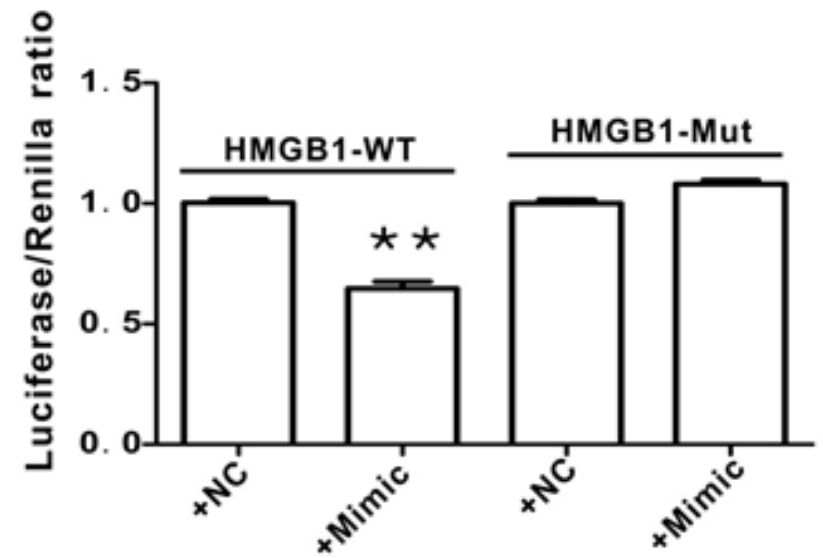

Figure 7. Transcriptional effect of the miR-181b-5p mimic on the $3^{\prime}$-UTR of HMGB1 in HAVSMCs. A) The 3'-UTR of wild type (WT) HMGB1 (the potential binding site for miR-181-5p is indicated in bold letters). B) The 3'-UTR of the mutated (MUT) HMGB1 (the MUT miR-181-5p binding site is indicated in bold letters). C) Luciferase activity in HAVSMCs in the presence of the miR-181b-5p mimic. At least three independent experiments were performed for each group.

Positive correlation between the plasma level of HMGB1 and blood pressure

HMGB1 plasma levels were significantly elevated in hypertensive patients compared with the healthy controls; this was dramatically reversed following treatment with ARB (Fig. 8C; $P<0.01$ ). As shown in Fig. 8D and E, there was a significant positive correlation between HMGB1 plasma levels and SBP $(\mathrm{r}=0.396, P<0.001)$ or DBP $(\mathrm{r}=0.885, P<0.001)$. 
A

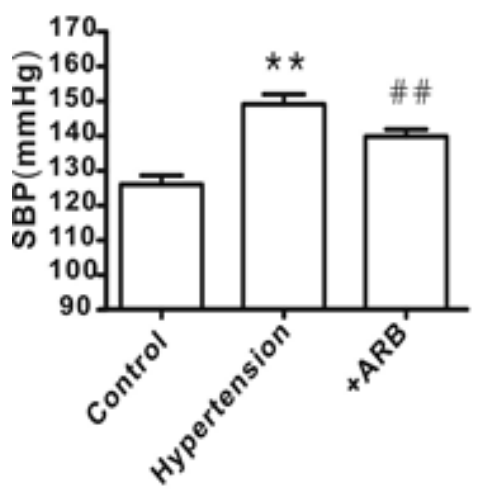

B

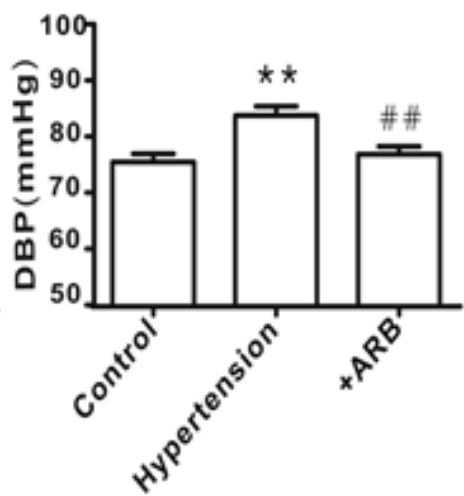

C

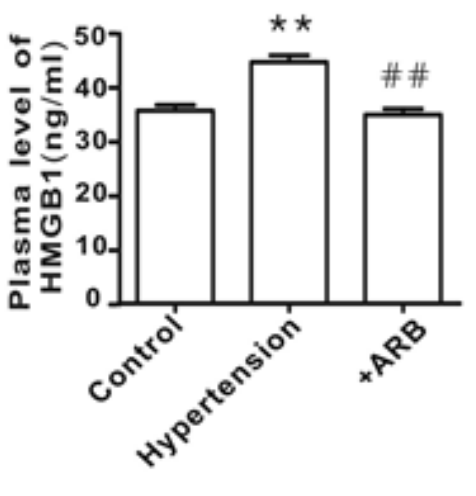

D

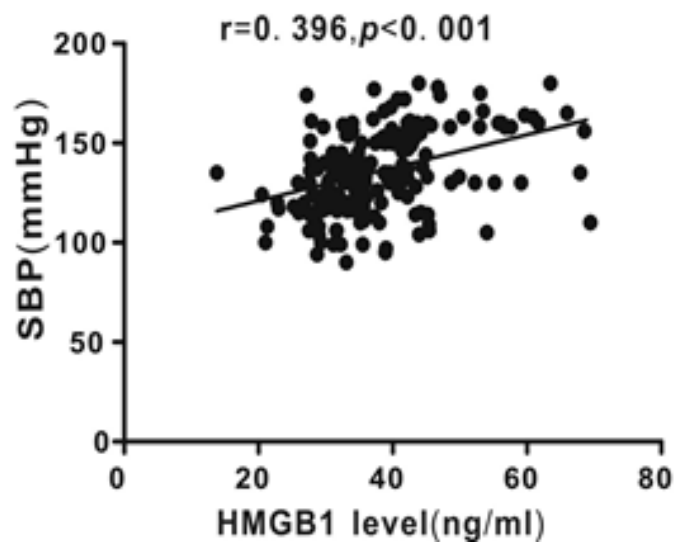

E

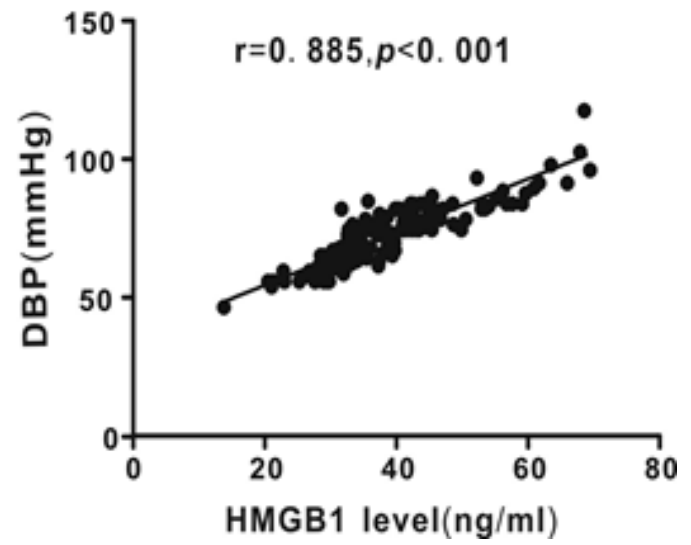

Figure 8. Correlation between HMGB1plasma levels and blood pressure. A and B) Subject blood pressure. C HMGB1plasma levels. D and E) Correlation between HMGB1 plasma levels of and blood pressure. Control group, $\mathrm{n}=58$; hypertension group, $\mathrm{n}=51 ;+\mathrm{ARB}$ treatment group, $\mathrm{n}=71$. ${ }^{* *} P<0.01$ vs. control, ${ }^{\#} P<0.01$ vs. hypertension.

\section{Clinical relevance of the miR-181b-5p/HMGB1 pathway in hypertension}

To evaluate the clinical relevance of the miR-181b$5 \mathrm{p} / \mathrm{HMGB} 1$ pathway in hypertension, the correlations between miR-181b-5p, HMGB1, Ang II, and intimamedia thickness (IMT, an index for carotid arterial remodeling) were analyzed. Consistent with the elevated HMGB1 levels in hypertension patients, the plasma level of miR-181b-5p was decreased concomitant with an increase in Ang II levels and IMT.These phenomena were attenuated in hypertensive patients following ARB treatment (Fig. 9A-C). There was a negative correlation between miR-181b-5p and HMGB1 (Fig. 9D), while a positive correlation was observed between Ang II and HMGB1 (Fig. 9E), IMT and HMGB1 (Fig. 9G), and IMT and Ang II (Fig. 9F).

\section{DISCUSSION}

In the present study, using the in vitro Ang II-induced phenotypic transformation model of vascular smooth muscle cells, we demonstrated that HMGB1 was upregulated, accompanied by down-regulation of contractile proteins and up-regulation of synthetic proteins. Both losartan and HMGB1 siRNA were able to attenuate Ang II-induced HAVSMC phenotypic transformation, migration, and proliferation. Bioinformatics analysis identified miR-181b-5p as an upstream regulator of Ang II-induced HMGB1 expression; miR-181b-5p mimic produced similar results to losartan and HMGB1 siRNA. The results of the dual luciferase reporter gene assay demonstrated the direct interaction between miR-181b-5p and HMGB1. The findings of the clinical study showed that the plasma level of miR-181b-5p was decreased, 
while HMGB1 and Ang II levels were increased in hypertensive patient's concomitant with an increase in IMT, a vascular remodeling index. These phenomena were attenuated in patients who received angiotensin receptor blockers. There was a negative correlation between the plasma levels of miR-181b-5p and HMGB1.
To the best of our knowledge, this is the first study to provide evidence that the miR-181b-5p/HMGB1 pathway may contribute to the phenotypic transformation of vascular smooth muscle cells and vascular remodeling in hypertensive patients.

Table 4. Baseline characteristics of participants.

\begin{tabular}{cccc}
\hline & $\begin{array}{c}\text { Control } \\
(\mathbf{n = 5 8})\end{array}$ & $\begin{array}{c}\text { Hypertension } \\
(\mathbf{n = 5 3})\end{array}$ & $\begin{array}{c}\text { +ARB } \\
(\mathbf{n}=\mathbf{7 1})\end{array}$ \\
\hline Age, yrs & $59.28 \pm 1.47$ & $60.83 \pm 1.43$ & $61.48 \pm 1.32$ \\
Male $(\%$ of total) & $29(15.9 \%)$ & $27(14.8 \%)$ & $34(18.7 \%)$ \\
Female $(\%$ of total) & $29(15.9 \%)$ & $26(14.3 \%)$ & $37(20.7 \%)$ \\
SBP(mmHg) & $124.36 \pm 2.48$ & $146.42 \pm 2.78^{* *}$ & $137.35 \pm 1.98^{\# \#}$ \\
DBP(mmHg) & $74.24 \pm 1.35$ & $82.04 \pm 1.51^{* *}$ & $75.54 \pm 1.32^{\# \#}$ \\
FBG, mmol/L & $5.49 \pm 0.07$ & $5.53 \pm 0.08$ & $5.50 \pm 0.08$ \\
TG, mmol/L & $1.46 \pm 0.08$ & $1.50 \pm 0.80$ & $1.56 \pm 0.08$ \\
TC, mmol/L & $5.14 \pm 0.11$ & $5.31 \pm 0.10$ & $5.33 \pm 0.08$ \\
HDL, mmol/L & $1.38 \pm 0.04$ & $1.27 \pm 0.05$ & $1.35 \pm 0.05$ \\
LDL, mmol/L & $3.09 \pm 0.12$ & $2.64 \pm 0.11$ & $2.84 \pm 0.29$ \\
ALT, U/L & $26.02 \pm 1.55$ & $26.35 \pm 2.48$ & $26.99 \pm 2.38$ \\
AST, U/L & $22.50 \pm 1.02$ & $22.99 \pm 1.11$ & $23.60 \pm 1.17$ \\
BUN, mmol/L & $5.24 \pm 0.17$ & $5.29 \pm 0.21$ & $5.62 \pm 0.14$ \\
UA, mmol/L & $348.97 \pm 11.53$ & $349.92 \pm 12.90$ & $355.41 \pm 11.26$ \\
Cr, $\mu \mathrm{mol} / \mathrm{L}$ & $84.07 \pm 1.22$ & $85.19 \pm 1.07$ & $85.75 \pm 0.81$ \\
\hline
\end{tabular}

Data presented as mean \pm SEM. $* * P<0.01$ vs. control, ${ }^{\# \#} P<0.01$ vs. hypertension. Abbreviations: Control for the healthy control people $(n=58)$; hypertension for the essential hypertensive patients without any drugs $(n=53)$; +ARB, the essential hypertensive patients only with angiotensin II receptor blockage for treatment $(n=71)$; SBP, systolic blood pressure; DBP, diastolic blood pressure; FBG, fasting blood glucose; TG, Triglyceride; TC, total cholesterol; HDL-C, high density lipoprotein cholesterol; LDL-C, low density lipoprotein cholesterol; ALT, Alanine transaminase; AST, Aspartate transaminase; BUN, blood urea nitrogen; UA, uric acid; Cr, creatinine.

Vascular remodeling in hypertension may initially be adaptive; however, eventually it becomes maladaptive and contributes to the development and complications of hypertension[28, 30].VSMCs phenotypic transformation is a major initiating factor of vascular remodeling in hypertension[10]. Under pathological conditions, VSMCs accelerate the phenotypic transformation from contractile to synthetic phenotype. This phenotypic switching obviously changes the functions of VSMCs[31]. For example, synthetic VSMCs migrate and proliferate more easily than contractile VSMCs. Furthermore, synthetic VSMCs can synthesize up 25 to 46 -fold more collagen than contractile VSMCs [11,32]; all of which are closely related to vascular remodeling in hypertension.

It is well known that the renin-angiotensin system is activated in most cases of essential hypertension and plays a key role in blood pressure elevation[33]. In essential hypertension, Ang II plasma levels are usually elevated.
Thus, ACEI and angiotensin receptor blockers constitute first-line treatments of anti-hypertension. There is evidence that Ang II is able to induce VSMCs phenotypic transformation $[34,35]$, which increases the proliferation and migration ability of VSMCs, resulting in vascular remodeling $[32,36]$. Consistent with these reports, the results of the present study demonstrated a downregulation of contractile proteins and an up-regulation of synthetic proteins in Ang II-treated HAVSMCs, concomitant with an increase in cellular migration and proliferation. These phenomena were attenuated in the presence of losartan, indicating that the in vitro model of Ang II-induced VSMCs phenotypic transformation was successful.

Hypertension has been described as an inflammatory disease and vascular inflammation is considered to play a critical role in vascular remodeling in several vascular diseases such as hypertension and atherosclerosis[37]. 
Both in vivo and in vitro studies have demonstrated that HMGB1 is critical for the development of vascular inflammation [36, 38, 39]. In addition, Ang II has also been shown to be involved in vascular inflammation [31, 40, 41]. However, whether there is a correlation between Ang II and HMGB1 unkown. In the present study, we found that HMGB1 expression (mRNA and protein) was significantly up-regulated in Ang II-treated HAVSMCs and that this was reversed in the presence of losartan, confirming a link between Ang II and HMGB1. As
HMGB1 plays an important role in many pathogenic processes, including VSMCs abnormal proliferation and migration[42], we hypothesize that HMGB1 has a key role in mediating Ang II-induced VSMCs phenotypic transformation, cellular migration, and proliferation. The results of this study demonstrate that knockdown of HMGB1 dramatically attenuated Ang II-induced phenotypic transformation of HAVSMCs, cellular migration, and proliferation, supporting our hypothesis.
A
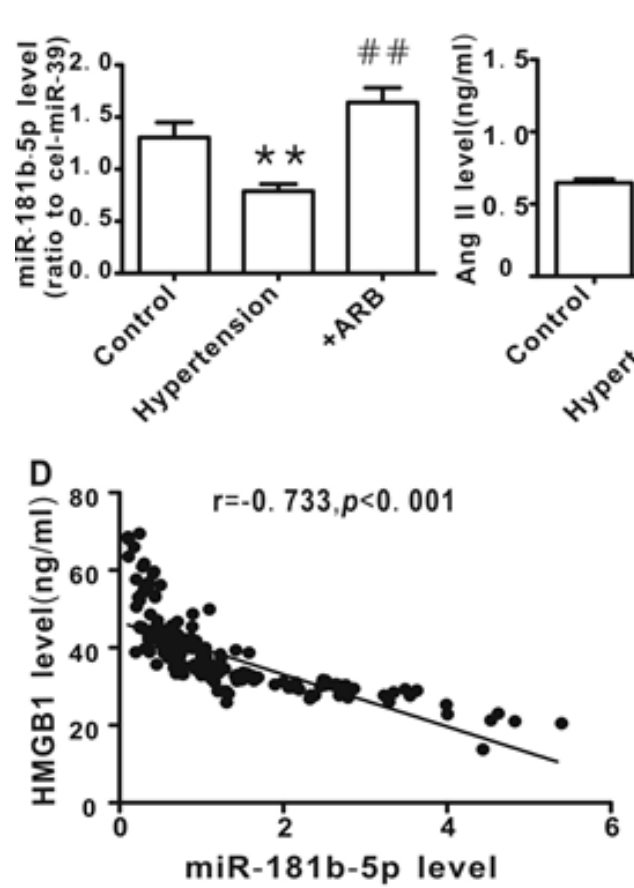

$\mathbf{F}$

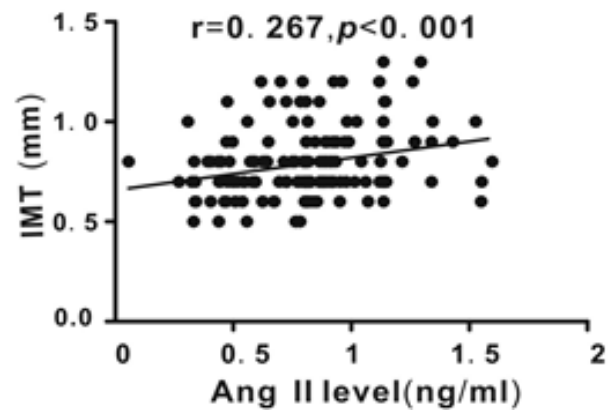

C

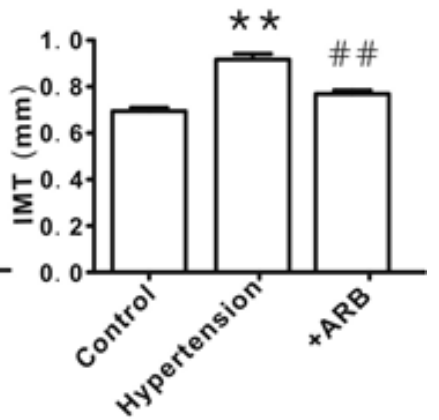

E

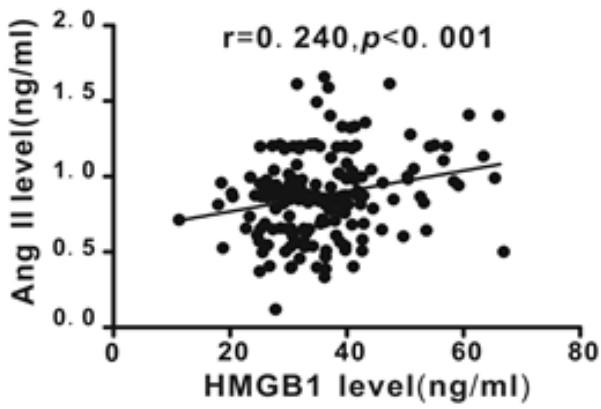

G

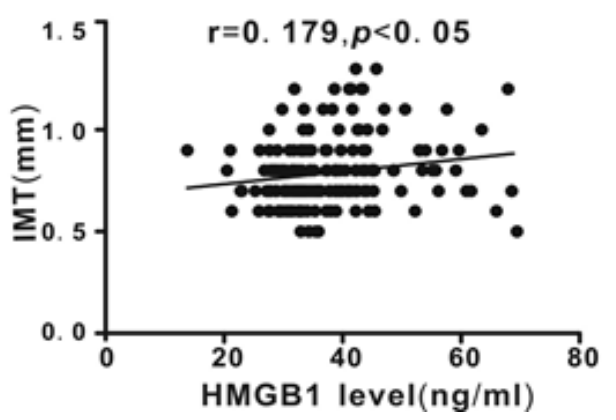

Figure 9. Correlation between miR-181b-5p, HMGB1, Ang II, and intima-media thickness (IMT) in hypertensive patients. A) miR-181b-5p plasma levels. B) Ang II plasma levels. C. IMT values, determined by carotid artery ultrasound. D) The correlation between HMGB1 level and miR-181b-5p level. E) The correlation between HMGB1 level and Ang II level. E) The correlation between IMT and Ang II level. F) The correlation between IMT and HMGB1 level. Control group, $\mathrm{n}=58$; Hypertension group, $\mathrm{n}=51$, Hypertension $+\mathrm{ARB}$ group, $\mathrm{n}=71$. ${ }^{*} * P<0.01$ vs. control, ${ }^{\#} P<0.01$ vs. Hypertension. 
To elucidate the mechanisms responsible for the upregulation of HMGB1 in Ang II-treated HAVSMCs, we used bioinformatics software to predict potential miRNAs targeting HMGB1; three candidate miRNAs (miR-103a3p, miR-107, and miR-181b-5p) were identified. In Ang II-treated HAVSMCs, only miR-181b-5p was dramatically down-regulated; this effect was reversed in the presence of losartan, suggesting that miR-181b-5p might be an upstream negative regulator of HMGB1. Using miR-181b-5p mimic, we found that HMGB1 mRNA and protein expression was suppressed in Ang IItreated HAVSMCs, confirming the role of miR-181b-5p in the suppression of HMGB 1expression. The results of the dual luciferase reporter gene assay indicated a direct interaction between miR-181b-5p and HMGB1.

To extend the clinical relevance of our cell studybased findings, we carried out a clinical study. Our results showed that the plasma levels were significantly elevated in hypertensive patient's concomitant with an increased vascular remodeling index(IMT), demonstrating a positive correlation between HMGB1 levels and IMT or blood pressure. These results suggest that HMGB1 may also contribute to vascular remodeling in essential hypertension. Interestingly, a number of studies have reported that plasma levels of HMGB1 were elevated in pulmonary arterial hypertension $(\mathrm{PAH})$ and contributed to the vascular remodeling of PAH [40, 43, 44]. Our current results indicate that HMGB1 has the same function in essential hypertension as in PAH. Our cell study demonstrated that HMGB1 mediated Ang II-induced phenotypic transformation of HAVSMCs. Moreover, we found a positive correlation between the plasma levels of HMGB1 and Ang II. Following treatment with an angiotensin receptor blocker, the plasma levels of HMGB1 in hypertensive patients were obviously reduced, confirming a link between Ang II and HMGB1. Finally, we examined the plasma levels of miR-181b-5p in hypertensive patients. The results showed that miR-181b$5 \mathrm{p}$ levels were markedly decreased and inversely correlated with the plasma levels of HMGB1. This decrease in miR-181b-5p levels accompanied by an increase in HMGB1 was reversed in hypertensive patients treated with angiotensin receptor blockers, further verifying the function of miR-181b-5p as a negative regulator of HMGB1. Of note, although the clinical study limited us to examining the expression of miR-181b-5p, HMGB1, $\alpha$-SMA, SM22 $\alpha$, and osteopontin in the blood vessels, it still supports our in vitro study findings.

In summary, the results presented here demonstrate for the first time that HMGB1 is up-regulated in essential hypertension, which contributes to vascular remodeling by modulating the phenotypic transformation of smooth muscle cells from contractile to synthetic type under the activation of the renin-angiotensin system. We have also identified miR-181b-5p as a negative regulator of HMGB1 expression. Thus, Ang II/ miR-181b-5p/ HMGB1 might form a novel pathway involved in the regulation of vascular remodeling in hypertension. Interfering with this pathway may have therapeutic and preventive value for hypertension.

\section{Limitation of the study}

This study had a major limitation that needs to be acknowledged and addressed. Although the miR-181b5p/HMGB1 pathway was shown to be involved in Ang IIinduced phenotypic transformation of smooth muscle cell in vitro, the clinical study limited verification of the involvement of the miR-181b-5p/HMGB1 pathway in phenotypic transformation of smooth muscle cells to the blood vessels of hypertensive patients. These issues will be solved using proper hypertension animal models in our future studies.

\section{Acknowledgements}

This work was supported by the National Natural Science Foundation of China (No. 81570334 to Tian-Lun Yang; No. 81573430 to Xiu-Ju Luo; and No.81373409 to Jun Peng); the Great Physician Foundation of Xiangya Hospital, Central South University, Changsha, China; and the Doctoral Independent Innovation Fund of the Central South University, Changsha, China (No. 2016zzts128 to Feng-Juan Li).

\section{Conflicts of interest}

The authors confirm that there are no conflicts of interest.

\section{References}

[1] Mills KT, Bundy JD, Kelly TN, Reed JE, Kearney PM, Reynolds K, et al. (2016). Global Disparities of Hypertension Prevalence and Control: A Systematic Analysis of Population-Based Studies From 90 Countries. Circulation, 134:441-450.

[2] Li W, Gu H, Teo KK, Bo J, Wang Y, Yang J, et al. (2016). Hypertension prevalence, awareness, treatment, and control in 115 rural and urban communities involving 47000 people from China. Journal of Hypertension, 34:39-46.

[3] Lewington S, Lacey B, Clarke R, Guo Y, Kong XL, Yang L, et al. (2016). The Burden of Hypertension and Associated Risk for Cardiovascular Mortality in China. JAMA Intern Med, 176:524-532.

[4] Chen WW, Gao RL, Liu LS, Zhu ML, Wang W, Wang YJ, et al. (2017). China cardiovascular diseases report 2015: a summary. J Geriatr Cardiol, 14:1-10.

[5] Hernanz R, Martinez-Revelles S, Palacios R, Martin A, Cachofeiro V, Aguado A, et al. (2015). Toll-like 
receptor 4 contributes to vascular remodelling and endothelial dysfunction in angiotensin II-induced hypertension. Br J Pharmacol, 172:3159-3176.

[6] Wu X, Fan R (2017). Identifications of potential therapeutic targets and drugs in angiotensin II-induced hypertension. Medicine (Baltimore), 96:e8501.

[7] Muñoz-Durango N, Fuentes C, Castillo A, GonzálezGómez L, Vecchiola A, Fardella C, et al. (2016). Role of the Renin-Angiotensin-Aldosterone System beyond Blood Pressure Regulation: Molecular and Cellular Mechanisms Involved in End-Organ Damage during Arterial Hypertension. International Journal of Molecular Sciences, 17:797.

[8] Schiffrin EL (2012). Vascular remodeling in hypertension: mechanisms and treatment. Hypertension, 59:367-374.

[9] Lu Y, Zhang L, Liao X, Sangwung P, Prosdocimo DA, Zhou G, et al. (2013). Kruppel-like factor 15 is critical for vascular inflammation. J Clin Invest, 123:42324241.

[10] Davis-Dusenbery BN, Wu C, Hata A (2011). Micromanaging vascular smooth muscle cell differentiation and phenotypic modulation. Arterioscler Thromb Vasc Biol, 31:2370-2377.

[11] Lacolley P, Regnault V, Nicoletti A, Li Z, Michel JB (2012). The vascular smooth muscle cell in arterial pathology: a cell that can take on multiple roles. Cardiovasc Res, 95:194-204.

[12] Shi N, Chen SY (2014). Mechanisms simultaneously regulate smooth muscle proliferation and differentiation. J Biomed Res, 28:40-46.

[13] Gomolak JR, Didion SP (2014). A role for innate immunity in the development of hypertension. Med Hypotheses, 83:640-643.

[14] Caillon A, Schiffrin EL (2016). Role of Inflammation and Immunity in Hypertension: Recent Epidemiological, Laboratory, and Clinical Evidence. Curr Hypertens Rep, 18:21.

[15] Marchesi C, Paradis P, Schiffrin EL (2008). Role of the renin-angiotensin system in vascular inflammation. Trends Pharmacol Sci, 29:367-374.

[16] Zhou C, Huang J, Chen J, Lai J, Zhu F, Xu X, et al. (2016). CYP2J2-Derived EETs Attenuated Angiotensin II-Induced Adventitial Remodeling via Reduced Inflammatory Response. Cell Physiol Biochem, 39:721-739.

[17] Meng Y, Chen C, Tian C, Du J, Li HH (2015). Angiotensin II-induced Egr-1 expression is suppressed by peroxisome proliferator-activated receptor-gamma ligand 15d-PGJ(2) in macrophages. Cell Physiol Biochem, 35:689-698.

[18] Scaffidi P, Misteli T, Bianchi ME (2002). Release of chromatin protein HMGB1 by necrotic cells triggers inflammation. Nature, 418:191-195.

[19] Li W, Sama AE, Wang H (2006). Role of HMGB1 in cardiovascular diseases. Current Opinion in Pharmacology, 6:130.

[20] Lange SS, Mitchell DL, Vasquez KM (2008). High mobility group protein B1 enhances DNA repair and chromatin modification after DNA damage. Proc Natl
Acad Sci U S A, 105:10320-10325.

[21] Porto A, Palumbo R, Pieroni M, Aprigliano G, Chiesa $\mathrm{R}$, Sanvito F, et al. (2006). Smooth muscle cells in human atherosclerotic plaques secrete and proliferate in response to high mobility group box 1 protein. FASEB J, 20:2565-2566.

[22] Yang J, Chen L, Yang J, Ding J, Rong H, Dong W, et al. (2012). High mobility group box-1 induces migration of vascular smooth muscle cells via TLR4dependent PI3K/Akt pathway activation. Mol Biol Rep, 39:3361-3367.

[23] Yang J, Chen L, Ding J, Fan Z, Li S, Wu H, et al. (2016). MicroRNA-24 inhibits high glucose-induced vascular smooth muscle cell proliferation and migration by targeting HMGB1. Gene, 586:268-273. Neth P, Nazari-Jahantigh M, Schober A, Weber C (2013). MicroRNAs in flow-dependent vascular remodelling. Cardiovasc Res, 99:294-303.

[25] Wang H, Jiang M, Xu Z, Huang H, Gong P, Zhu H, et al. (2015). miR-146b-5p promotes VSMC proliferation and migration. Int J Clin Exp Pathol, 8:12901-12907.

[26] Hartmann P, Schober A, Weber C (2015). Chemokines and microRNAs in atherosclerosis. Cell Mol Life Sci, 72:3253-3266.

[27] Pahl MC, Kimberly D, Gabor G, Irene H, Elmore JR, Schworer CM, et al. (2012). MicroRNA expression signature in human abdominal aortic aneurysms. Bmc Medical Genomics, 5:25.

[28] Sun HJ, Liu TY, Zhang F, Xiong XQ, Wang JJ, Chen $\mathrm{Q}$, et al. (2015). Salusin-beta contributes to vascular remodeling associated with hypertension via promoting vascular smooth muscle cell proliferation and vascular fibrosis. Biochim Biophys Acta, 1852:1709-1718.

[29] Perge P, Decmann A, Pezzani R, Bancos I, Fassina A, Luconi M, et al. (2018). Analysis of circulating extracellular vesicle-associated microRNAs in cortisol-producing adrenocortical tumors. Endocrine, 59:280-287.

[30] Calhoun DA, White WB, Krum H, Guo W, Bermann G, Trapani A, et al. (2011). Effects of a novel aldosterone synthase inhibitor for treatment of primary hypertension: results of a randomized, double-blind, placebo- and active-controlled phase 2 trial. Circulation, 124:1945-1955.

[31] Zhang L, Xie P, Wang J, Yang Q, Fang C, Zhou S, et al. (2010). Impaired peroxisome proliferator-activated receptor-gamma contributes to phenotypic modulation of vascular smooth muscle cells during hypertension. J Biol Chem, 285:13666-13677.

[32] Doran AC, Meller N, McNamara CA (2008). Role of smooth muscle cells in the initiation and early progression of atherosclerosis. Arterioscler Thromb Vasc Biol, 28:812-819.

[33] Tamura K, Kanaoka T, Kobayashi R, Ohki K, Ohsawa M (2015). TLR4 as a possible key regulator of pathological vascular remodeling by Ang II receptor activation. Hypertens Res, 38:642-643.

[34] Ren XS, Tong Y, Ling L, Chen D, Sun HJ, Zhou H, et 
al. (2017). NLRP3 Gene Deletion Attenuates Angiotensin II-Induced Phenotypic Transformation of Vascular Smooth Muscle Cells and Vascular Remodeling. Cell Physiol Biochem, 44:2269-2280.

[35] Savoia C, Burger D, Nishigaki N, Montezano A, Touyz RM (2011). Angiotensin II and the vascular phenotype in hypertension. Expert Rev Mol Med, 13:e11.

[36] Te Riet L, van Esch JH, Roks AJ, van den Meiracker AH, Danser AH (2015). Hypertension: reninangiotensin-aldosterone system alterations. Circ Res, 116:960-975.

[37] Dange RB, Agarwal D, Teruyama R, Francis J (2015). Toll-like receptor 4 inhibition within the paraventricular nucleus attenuates blood pressure and inflammatory response in a genetic model of hypertension. J Neuroinflammation, 12:31.

[38] Mitchell GF (2014). Arterial stiffness and hypertension: chicken or egg? Hypertension, 64:210214.

[39] Siti HN, Kamisah Y, Kamsiah J (2015). The role of oxidative stress, antioxidants and vascular inflammation in cardiovascular disease (a review). Vascul Pharmacol, 71:40-56.

[40] Jia D, He Y, Zhu Q, Liu H, Zuo C, Chen G, et al. (2017). RAGE-mediated extracellular matrix proteins accumulation exacerbates HySu-induced pulmonary hypertension. Cardiovasc Res, 113:586-597.

[41] Wang M, Kim SH, Monticone RE, Lakatta EG (2015). Matrix metalloproteinases promote arterial remodeling in aging, hypertension, and atherosclerosis. Hypertension, 65:698-703.

[42] Nogueira-Ferreira R, Ferreira-Pinto MJ, Silva AF, Vitorino R, Justino J, Costa R, et al. (2017). HMGB1 down-regulation mediates terameprocol vascular antiproliferative effect in experimental pulmonary hypertension. J Cell Physiol, 232:3128-3138.

[43] Huang YY, Su W, Zhu ZW, Tang L, Hu XQ, Zhou SH, et al. (2016). Elevated serum HMGB1 in pulmonary arterial hypertension secondary to congenital heart disease. Vascul Pharmacol, 85:66-72.

[44] Li WJ, Hu K, Yang JP, Xu XY, Li N, Wen ZP, et al. (2017). HMGB1 affects the development of pulmonary arterial hypertension via RAGE. Eur Rev Med Pharmacol Sci, 21:3950-3958. 